\title{
METILFENIDATO: USO INDISCRIMINADO
}

\section{METHYLFENIDATE: INDISCRIMINATED USE}

\section{Kezia Gonçalves Rocha;}

Bacharel em Farmácia. Alfa - Faculdade de Almenara, Almenara-MG Brasil.

\section{Gabriel Barbosa A. Silva}

Bacharel em Farmácia. Alfa - Faculdade de Almenara, Almenara-MG

Brasil.

\section{Luiza Gobira Lacerda;}

Especialista. Alfa - Faculdade de Almenara, Almenara-MG Brasil.

Viviane Amaral Toledo Coelho Doutora. vivianeatc@yahoo.com.br. Almenara - MG

Ronivaldo Ferreira Mendes Mestre em Educação. - IFNMG - Campus Almenara, Almenara-MG. Brasil

\section{RESUMO}

Objetivo: O objetivo desse artigo é analisar os perigos do uso indiscriminado do metilfenidato por jovens estudantes universitários não diagnosticados com TDAH que se automedicam em busca de melhoras cognitivas e concentração nos estudos, abordando também a diligência do profissional farmacêutico na conjuntura desse cenário. Metodologia: Trata-se de uma revisão sistemática da literatura, de caráter descritivo, qualitativo, no qual foi executado o levantamento bibliográfico de artigos científicos publicados entre os anos de 2008 a 2018, tendo entre eles apenas uma 
referência equivalente ao ano de 1998 que corresponde à Portaria SVS/MS no 344, de 12 de maio de 1998. Com base nas fontes de dados eletrônicos: Scientific Eletronic Library Online (Scielo), Portal Regional BVS e Google Acadêmico, no qual foram utilizados os seguintes Descritores em Ciências da Saúde (DeCS): "metilfenidato"; "uso terapêutico", "efeitos adversos", "universitários" e "atenção farmacêutica". Resultados: Parte considerável dos consumidores de metilfenidato utiliza o medicamento com a finalidade de melhorar seu desempenho acadêmico ou aprimoramento cognitivo. Aumentando o consumo no decorrer do curso. 66,67\% dos acadêmicos que utilizam essa substância obtiveram por meio de doação de amigos. Observar uma taxa maior de uso presente no sexo feminino, com $61,90 \%$, aproximadamente $68,25 \%$ entre jovens com idade entre 18-23 anos. A Atenção Farmacêutica realizada de forma sistematizada e documentada pode identifica ou resolve os problemas farmacoterapêuticos e possivelmente o uso indiscriminado do metilfenidato. Considerações finais: Desta forma as informações se consolidariam nos saberes adquiridos, possibilitaria a formação de proficionais com uma visão crítica apurada, conduta profissional e ações, transformadoras da sociedade.

Palavras-chave: Metilfenidato. Uso terapêutico. Efeitos adversos. Universitários. Atenção farmacêutica.

\section{ABSTRACT}

Objective: The aim of this paper is to analyze the dangers of indiscriminate use of methylphenidate by young undiagnosed ADHD college students who are selfmedicating in pursuit of cognitive improvement and concentration in their studies, and also addressing the diligence of pharmacists in this scenario. Methodology: This is a systematic, descriptive and qualitative literature review, in which a bibliographic survey of scientific articles published between 2008 and 2018 was performed, having only one reference equivalent to the year 1998 that corresponds SVS / MS Ordinance No. 344 of May 12, 1998. Based on electronic data sources: Scientific Electronic Library Online (Scielo), VHL Regional Portal and Google Scholar, in which the following Health 
Sciences Descriptors ( DeCS): "methylphenidate"; "Therapeutic use" and "adverse effects" "university" "pharmaceutical attention". Results: A considerable proportion of methylphenidate users use the drug in order to improve their academic performance or cognitive enhancement. Increasing consumption over the course. $66.67 \%$ of academics who use this substance obtained through donation of friends. Observe a higher rate of use present in females, with $61.90 \%$, approximately $68.25 \%$ among young people aged 18-23 years. Pharmaceutical Care carried out in a systematic and documented manner can identify or solve pharmacotherapeutic problems and possibly the indiscriminate use of methylphenidate. Final considerations: In this way the information would be consolidated in the acquired knowledge, would enable the formation of professionals with an accurate critical vision, professional conduct and actions, transforming the society.

Keywords: Methylphenidate. Therapeutic use. Adverse effects. College students. Pharmaceutical attention.

\section{INTRODUÇÃO}

Os psicoestimulantes ou estimulantes cerebrais são substâncias que possui a capacidade de elevar as atividades cerebrais melhorando o desempenho cognitivo, concentração e memória, modificando ou corrigindo a transmissão neuroquímica em áreas do Sistema Nervoso que regulam o estado do humor (o nível da vitalidade, energia, emoções e a variação entre alegria e tristeza). As substâncias mais utilizadas para esse proposito são: o metilfenidato, cafeína, modafinil, piracetam, bebidas energéticas e anfetaminas (MORGAN et al., 2016).

Neste panorama, o consumo de medicamentos estimulantes cerebrais aumentou significativamente, devido ao fortalecimento do paradigma biomédico, ao crescimento da indústria farmacêutica, à ampliação do acesso aos medicamentos e à intensificação dos processos de mercantilização da saúde e medicalização da sociedade, na qual jovens e adultos fazem uso para melhorar o desempenho cognitivo ou para fins recreativos (CALIMAN; RODRIGUES, 2014). 
É nesse contexto que o metilfenidato, entra em destaque, sendo um dos psicofármacos mais utilizados na psiquiatria infantil, receitado para o tratamento do Transtorno de Déficit de Atenção e Hiperatividade (TDAH), mas que frequentemente está sendo utilizado de forma indiscriminada por jovens acadêmicos e universitário a fim de adquirirem melhora cognitiva, concentração e memória (SILVA et al., 2012).

Em consonância a estas conceituações, o mecanismo de ação desta substância consiste em estimular o Sistema Nervoso Central, por ser um potente inibidor da recaptação de dopamina e de noradrenalina na fenda sináptica, ocorre um aumento dos seus níveis extracelulares. Sendo assim, o fármaco eleva o nível de alerta e incrementa os mecanismos excitatórios do cérebro, o que resulta em uma melhor concentração, coordenação motora e controle dos impulsos (COLI; SILVA; NAKASU, 2016).

De acordo com a Portaria $n^{\circ} 344$, de 12 de maio de 1998 que aprova o regulamento sobre substâncias sujeitas a controle especial, o metilfenidato só pode ser dispensado mediante retenção da notificação de receita, onde o metilfenidato está incluso na lista "A3" (substâncias psicotrópicas), sujeito a notificação de receita "A" (notificação de receita amarela).

Estudos recentes apontam que, entre os anos de 2003 e 2012, o consumo de metilfenidato aumentou $775 \%$ no Brasil. Tal crescimento expressivo pode ser explicado pela ampliação dos critérios diagnósticos para o Transtorno de Déficit de Atenção e Hiperatividade (TDAH), elevando o número de usuários em potencial e facilitando o acesso ao fármaco (COLI; SILVA; NAKASU, 2016).

Segundo Fardini e Piloto (2015) atualmente estudantes e profissionais buscam meios para aumentar seu poder produtivo, estudar exageradamente sem se cansar e principalmente lembrar o que estudou. Devido isso o metilfenidato está sendo amplamente utilizado por indivíduos não portadores de TDAH com a finalidade de melhorar a concentração e aumentar o desempenho intelectual.

O uso de Substâncias Psicoativas (SPAs) entre universitários tem sido amplamente divulgado e debatido já que envolve interesses econômicos da indústria farmacêutica, bem como questões éticas e legais. A universidade é um ambiente que 
exige níveis elevados de dedicação e trabalho, e cada estudante manipula diferentemente a carga de estresse imposta.

O objetivo deste artigo foi analisar os perigos do uso indiscriminado do metilfenidato por jovens estudantes universitários não diagnosticados com TDAH que se automedicam em busca de melhoras cognitivas e concentração nos estudos, abordando também a diligência do profissional farmacêutico na conjuntura desse cenário.

\section{METODOLOGIA}

Trata-se de uma revisão sistemática da literatura, de caráter descritivo, qualitativo, com a seguinte questão norteadora: atualmente aborda-se nos meios de comunicação sobre a "pílula da inteligência" (metilfenidato), não obstante, o que os estudos científicos têm apontado sobre a motivação, expectativa, efeitos e prejuízos do uso indiscriminado de metilfenidato por estudantes universitários em todo o mundo?

Diante da pergunta, foi executado o levantamento bibliográfico de artigos científicos publicados entre os anos de 2008 a 2018, tendo entre eles apenas uma referência equivalente ao ano de 1998 que corresponde à Portaria SVS/MS nº 344, de 12 de maio de 1998 que discorre a exigência da prescrição em receita amarela para a dispensação do metilfenidato. Após a fase de identificação os conteúdos foram revisados, por meio de leitura seletiva, para se definir quais representaria a etapa determinante para escolha do material que consisti em fonte bibliográfica para a pesquisa.

A pesquisa foi norteada pelas bases de dados eletrônicos: Scientific Eletronic Library Online (Scielo), Portal Regional BVS e Google Acadêmico. Para critérios de inclusão dos artigos resultantes foram adotados parâmetros como; relevância ao tema, artigos científicos e estudos escritos em português e inglês, atualidade das informações, ou conteúdos de anos anteriores se pertinentes. Foram utilizados os seguintes Descritores em Ciências da Saúde (DeCS): "metilfenidato"; "uso terapêutico" "efeitos adversos" "universitários" e " atenção farmacêutica". 
Desta forma, abrangeu-se um total de 24 conteúdos estudados, entre eles uma monografia onde através da leitura seletiva e minuciosa foram antepostos oito artigos que apresentassem em seu conteúdo especificações sobre o metilfenidato. E os demais como base destes estudos, sendo eles os mais utilizados no decorrer da presente revisão bibliográfica, selecionados para compor a descrição dos resultados e discussão.

TRANSTORNO DE DÉFICIT DE ATENÇÃO COM HIPERATIVIDADE (TDAH)

O (TDAH), também chamado de Síndrome Hipercinética, é caracterizado como uma desordem comportamental que tem origem na infância, tendo características essenciais como: sinais de impulsividade, hiperatividade ou inquietude e desatenção inconsistente com o nível de desenvolvimento. De acordo com a Associação Brasileira de Déficit de Atenção o TDAH pode ser definido como um transtorno neurobiológico, de causas genéticas, que aparece na infância $e$ frequentemente acompanha o indivíduo por toda a sua vida, sendo mais predominante em indivíduos do sexo masculino (NEGREIROS; TELLES, 2009).

Pode-se observar que crianças e jovens com TDAH tem dificuldade com atividades de longa duração, que exijam concentração, apresenta alguns sinais como esquecimento, desorganização, não consegue ficar envolvida em uma única atividade, impaciente, impulsivo e não pensa antes de agir provocando situações perigosas, tornando-se agressivo nas suas relações sociais (MONTEIRO et al., 2017).

Estima-se que o número de indivíduos com diagnóstico de TDAH tem aumentado nos últimos anos. As estimativas de prevalência desse transtorno variam muito. Estudos epidemiológicos relatam que essa variação encontra-se entre 3\% e $16 \%$. No Brasil, as estimativas de predominância de TDAH em crianças e adolescentes também variam bastante, com valores entre 0,9\% e 26,8\%. Nos Estados Unidos, estimase que esse transtorno afete pelo menos 5 milhões de crianças com idades entre 4 e 17 anos (BRASIL, 2014). 
Diante do exposto ainda não é possível identificar marcadores biológicos para a realização do diagnóstico do TDAH, baseando-se fundamentalmente no quadro clínico comportamental do paciente, observado em pelo menos dois ambientes, por exemplo, o escolar e o domiciliar, por período mínimo de seis meses. $\mathrm{O}$ início pode ser observado a partir dos três anos, mas é apenas diagnosticável após a inserção da criança no ambiente escolar. Sendo assim o metilfenidato aparece como o medicamento de escolha para o tratamento das pessoas diagnósticas com TDAH. Desta forma, comportamentos tidos como socialmente inadequados pelos pais e educadores tornam-se justificativa de medicação (MONTEIRO et al., 2017).

\section{METILFENIDATO: "DROGA" DA INTELIGÊNCIA}

O metilfenidato é um agente estimulante moderado do sistema nervoso central (SNC) indicado como adjuvante a intervenções psicológicas, educacionais e sociais no tratamento de distúrbios de hiperatividade. Este medicamento foi patenteado em 1994, passou a ser comercializado como psicoestimulante leve e chegou ao mercado estadunidense no ano de 1956 e mais tarde no Canadá, por volta de 1979. A Agência Nacional de Vigilância Sanitária (ANVISA) autorizou a comercialização no Brasil apenas em 1998, e, desde então seu consumo no país tem sido cada vez mais crescente (CALAZANS; BELO, 2017).

O metilfenidato foi classificado pela convenção da ONU (Organização das Nações Unidas) como droga psicotrópica. Por outro lado, de acordo com a portaria SVS/MS n 344/98 foi classificado na lista A3 (substâncias psicotrópicas), mas sujeito a notificação de receita A. Assim seu uso necessita de controle especial por apresentar risco de abuso e dependência (COLI; SILVA; NAKASU, 2016).

O Metilfenidato é comercializado no Brasil com os nomes comerciais Ritalina ${ }^{\circ}$ Ritalina LA® e Concerta ${ }^{\circledR}$, administrados por via oral. O consumo desse fármaco para o aprimoramento cognitivo vem aumentando no Brasil e no mundo. Segundo o relatório da ONU sobre produção de psicotrópicos, sua produção mundial alcançou a marca de 
71.6 toneladas em 2013 e seu consumo global atingiu um novo nível recorde de 72 toneladas. Dentre os maiores consumidores o Brasil encontra-se na $5^{\circ}$ posição (GONÇALVES; RIBEIRO, 2018).

Elevados níveis de produtividade em curto prazo, com baixo custo e alta qualidade, são imperativos colocados pela ditadura do sucesso a qualquer custo. Com base neste que contexto o uso de anfetaminas como o metilfenidato, tem sido amplamente utilizado, em grande parte, por universitários, empresários e profissionais da área de saúde. Isso se dá devido estes usuários, terem maior conhecimento sobre a droga em relação à população (BRANT; CARVALHO, 2012).

Entre os efeitos que fármaco teria no aumento do desempenho cognitivo, destacam-se diminuição no sono que possibilita a pessoa estudar por mais tempo, o aumento da concentração e da vigília, melhor rendimento, maior produtividade e um levante da energia que ajuda a produzir mais. Esse efeito de aprimoramento do desempenho em pessoas com ou sem diagnóstico parece ser similar ao das anfetaminas, o que reforça o uso do metilfenidato por estudantes (GONÇALVES; RIBEIRO, 2018).

\section{MECANISMO DE AÇÃO DO METILFENIDATO E AS CONSEQUÊNCIAS DA UTILIZAÇÃO ABUSIVA}

No homem, o sistema nervoso central (SNC) é responsável por receber e processar informações. Essas informações são distribuídas através dos neurônios, células pelas quais os impulsos nervosos se propagam até que cheguem à célula alvo. A comunicação entre os neurônios é feita por meio de sinapses, predominantemente químicas, que fazem uso de neurotransmissores, substâncias químicas capazes de causar uma resposta. Os neurotransmissores agem sobre a célula receptora, e em circunstâncias normais cada tipo de informação requer determinados neurotransmissores para transmiti-la ao neurônio seguinte (ANDRADE et al., 2018).

Segundo o mesmo autor, a quantidade liberada dessas substâncias também é determinada pelo tipo de sinal a ser encaminhado. Diversas drogas agem justamente aumentando a liberação de neurotransmissores ou então deixando os neurônios mais 
receptivos a esses neurotransmissores. O metilfenidato age no sistema nervoso central inibindo a recaptação de dopamina neurotransmissor responsável pelo controle motor, e de noradrenalina neurotransmissor responsável pela excitação física, mental e de bom humor. Neste caso o fármaco impede que as catecolaminas sejam recapturadas pelas terminações nervosas, assim os neurotransmissores permanecem ativos por mais tempo no espaço sináptico dando ao indivíduo um aumento da concentração, coordenação motora e excitação.

Atualmente, não existem dados suficientes que permitam concluir que o uso de metilfenidato pode melhorar significativamente as habilidades cognitivas de indivíduos saudáveis, mesmo assim seu uso não prescrito tem sido uma prática em ascensão, principalmente entre os universitários (BARROS; ORTEGA, 2011). Uma possível explicação para o aumento nas taxas de prevalência desse uso é que os universitários parecem desconhecer os efeitos negativos, valorizando apenas os efeitos positivos. Há indícios de que o metilfenidato provoca efeitos similares aos provocados por SPAs (substâncias psicoativas) ilícitas, como a cocaína, causando sentimentos de euforia, estimulação e alerta, o que em longo prazo pode desencadear transtornos como paranoia e esquizofrenia (FERNANDES et al., 2017).

Os estudos demonstraram as seguintes reações adversas com o uso do metilfenidato: aumento discreto da frequência cardíaca, dor de cabeça, ansiedade, nervosismo, tontura, sonolência e insônia. Por conseguinte, tais efeitos foram pouco frequentes e bem tolerados. Contudo, a maioria dos estudos foi conduzida em curto prazo ou sob efeito de dose única impossibilitando, portanto, avaliar o desenvolvimento de dependência e tolerância (MONTEIRO et al., 2017).

Estudo com camundongos demonstrou que o uso de metilfenidato, em baixas doses foi capaz de melhorar a memória numa tarefa de condicionamento ao medo, porém, quando usado em altas doses tornou-se prejudicial a esse tipo de memória embora tenha melhorado a memória espacial. Tal achado reside no fato de que doses diferentes do metilfenidato foram capazes de promover potenciação de substratos neurais diferentes, que são por sua vez ativados em diferentes tipos de memória. Em seres humanos saudáveis os efeitos do metilfenidato também parecem ser doses 
dependentes. Doses mais elevadas (maiores do que as indicadas para o tratamento de TDAH) aumentam a atividade locomotora, prejudicam a atenção e a memória de trabalho (FERNANDES et al., 2017).

\section{RESULTADOS E DISCUSSÃO}

A presente pesquisa procurou investigar a interface entre a medicalização referida como "salvadores" e "resolução rápida dos problemas" de uma sociedade moderna que se caracteriza pela a expansão da influência médica e farmacológica e a busca do melhoramento cognitivo evidenciado principalmente em meios acadêmicos, na qual estudantes saudáveis especulam melhorar o processamento mental de informações ampliando a capacidade cerebral. Estudos indicam que o uso indiscriminado desse fármaco ocorre principalmente por acadêmicos da área da saúde em destaque para o curso de medicina dissemelhante da presente pesquisa que procurava investigar a diferença da amostra através da analise de discentes de diferentes cursos de graduação.

Wille e Salvi (2018) destaca a importância de um bom rendimento nos estudos principalmente pelo fato de que o mesmo acaba se tornando um passo muito importante para uma vida profissional de sucesso. Desta forma, pode-se entender que a grande frequência do uso do metilfenidato se da pela decorrência do ambiente competitivo associado a fatores estressante envolvendo a falta de tempo para estudos e cansaço mental. Resultado similar foi mencionado por Morgan et al. (2017) no qual 80\% dos usuários consideram o uso de estimulantes efetivos para o aumento da concentração e redução do sono.

Segundo Carneiro et al. (2013) o uso do metilfenidato aumenta com o decorrer do curso, tal explicação seria uma maior carga horária somada a uma maior quantidade de conteúdo. No entanto de acordo Coli et al. (2016) e Morgan et al. (2017) não há diferença significativa entre períodos. Outro fator que poderia estar relacionado a esse aumento no consumo seria a utilização de forma errônea e sem os devidos cuidados e 
orientações de um profissional habilitado, favorecendo o uso abusivo, com risco de dependência (AFFONSO et al., 2016).

Em relação à aquisição do medicamento segundo Andrade et al. (2018) estudantes relatam não ser difícil, evidências que estão de acordo Coli et al. (2016 ) no qual $66,67 \%$ dos acadêmicos que utilizam essa substância obtiveram por meio de doação de amigos. A pesquisa de Wille e Salvi (2018) indica que também é possível adquirir o fármaco pela internet sem a prescrição médica, já outros conseguindo o receituário declarando portador de TDAH, corroborando com as mesmas evidências apresentadas por AFFONSO et al. (2016).

Segundo Calazans et al. (2017) quando se trata do sexo dos estudantes pode se observar uma taxa de uso maior presente no sexo feminino, com $61,90 \%$, em relação ao sexo masculino com 38,10\%. Resultados semelhantes aos de Morgan et al. (2017), com 60,2\%. Porém dados demonstrados por Coli et al. (2016) entre os acadêmicos de uma Faculdade de Medicina do Sul de Minas Gerais houve uma diferença significativa de consumo entre os gêneros masculino e feminino, sendo os homens os maiores consumidores de metilfenidato. A prevalência de consumo segundo este mesmo estudo e de aproximadamente $68,25 \%$ entre jovens com idade entre $18-23$ anos, devido muitos jovens nessa faixa etária estarem se preparando para prestar vestibular ou muitos já estarem nas universidades.

Dos resultados obtidos por Correia et al. (2013) as pessoas que fazem o uso indiscriminado, 64,86\% informaram ter apresentado efeitos colaterais. Sendo mais frequentes: taquicardia e ansiedade seguidos de tremores, perda de apetite e boca seca respectivamente. Wille e Salvi (2018) apontam também que 61,54\% dos usuários já manifestaram algum tipo de reações adversas relacionadas ao fármaco, tais como: depressão pós-efeito, nervosismo, cansaço e sono excessivo. Outros efeitos adversos citados por Andrade et al. (2018) ocasionados pelo metilfenidato é acatisia, alteração do humor, insônia, sendo que seu uso a longo prazo pode ocasionar alucinações e dependência, dentre outros. Contudo de todos os estudantes que afirmaram usar o metilfenidato, menos da metade $(38,46 \%)$ relatou que não houve nenhuma ocorrência de efeitos adversos. 
Entretanto Moura (2017) já sistematiza os efeitos colaterais e os classificam como: os efeitos de curto prazo, onde o usuário pode apresentar anorexia, ansiedade, irritabilidade, labilidade emocional podendo ainda provocar cefaleia e dor abdominal. Com menor frequência, podem-se observar alterações de humor, tiques, pesadelos, isolamento social e, ainda mais raramente, psicose. Os efeitos de maior importância do metilfenidato considerados em longo prazo estão associados à dependência, efeitos cardiovasculares e possível redução da estatura.

Mota et al. (2008) Cita muito bem a importância da orientação farmacêutica principalmente em relação ao tempo adequado do tratamento para que o paciente não extrapole o tempo de uso do fármaco, podendo assim obter como consequência alguns efeitos indesejáveis.

Pois as consequências do uso do Metilfenidato parecem ser simples, mas no decorrer do tempo o usuário amplifica a dose. Aumentando assim a tolerância e consequentemente o consumo. Segundo estudos feitos no Brasil há evidências sobre o mau uso da medicação ainda que pouco difundido (MOURA 2017).

Embora o uso do metilfenidato esteja associado diretamente ao diagnóstico de TDAH, ela não é recomendada em todos os casos onde o transtorno é diagnosticado (ITABORAHY; ORTEGA, 2013).

Isso porque o mesmo pode desencadear outros problemas no caso a interação medicamentosa, um fator clínico em que os efeitos de um fármaco são alterados ou anulados pela presença de outro fármaco, alimento, bebida ou algum agente químico do ambiente (JACOMINI; SILVA, 2011).

Fernandes e Cembranelli (2015) expõe que a prática da atenção farmacêutica está relacionada ao acompanhamento farmacoterapêutico, pois um paciente bem orientado quanto o administração adequada das doses aumenta relativamente às chances de adesão ao tratamento cooperando positivamente para o uso racional dos medicamentos (URM) desta forma o paciente possui uma maior possibilidade de melhorar a sua qualidade de vida.

$\mathrm{Na}$ atenção farmacêutica é importante sempre informar as orientações e cuidados ao paciente, podendo deixar claro a necessidade do tratamento proposto. Através de 
uma fácil compreensão e linguagem acessível, de forma adequada quanto ao uso, adesão e aos cuidados relevantes ao medicamento (MOURA, 2017)

Segundo mesmo autor, a atenção farmacêutica pode diminuir o número de eventos adversos, aumentar a qualidade assistencial e baixar os custos hospitalares. Com a atenção e intervenções farmacêuticas, a probabilidade do uso racional de medicamentos se elevará, há relatos sobre esta atividade, principalmente em grupos especiais de pacientes.

Fernandes e Cembranelli (2015) alegam que através de uma orientação adequada a farmacoterapia pode ser maximizada, porém essa não e uma atribuição exclusiva do farmacêutico, mas devido o seu maior contato com o público e abrangência em conhecimento farmacológico dá a esse profissional uma oportunidade de contribuir grandemente no combate ao uso irracional de medicamentos.

Reis et al. (2018) aborda que a presença do farmacêutico para a análise das prescrições e monitoramento das receitas médicas e indispensável, pois essa prática assegura que a farmacoterapia seja de qualidade e os erros de prescrições, interações medicamentosas principalmente entre fármacos de controle especial como $\mathrm{o}$ metilfenidato pode ser minimizado. No presente momento, este problema ocorre por conta de diversos fatores, entre eles: a falta de atenção na anamnese do paciente por parte do médico, a procura de uma substância que tenha efeito momentâneo para o estímulo cognitivo dos estudantes ou até a facilidade de conseguir o fármaco sem prescrição médica.

Segundo Fernandes e Cembranelli (2015) o profissional farmacêutico pode ser entendido como um agente de saúde de fácil acesso e encontrado na maioria das farmácias e drogarias. A atuação desses profissionais pode favorecer a redução dos problemas relacionados à automedicação e o uso indiscriminado do metilfenidato. Tanto a utilização de medicamentos isentos de prescrição (MIPs) ou medicamentos que exigem um controle especial como o metilfenidato acaba se tornando um processo complexo com múltiplos determinantes envolventes. As diretrizes farmacoterápicas adequadas para a condição clínica do indivíduo são elementos essenciais para a determinação do emprego dos medicamentos. 


\section{CONSIDERAÇÕES FINAIS}

Considerando os resultados alcançados no desenvolvimento das atividades foi possível identificar que a predominância do uso indiscriminado do metilfenidato está relacionada a jovens acadêmicos que possuem o entendimento sobre os efeitos, na maioria das vezes "positivos" do fármaco, porem isso ocorre devido a grande quantidade de conteúdo a ser estudado em consonância a falta de tempo e influência de outros usuários que alegam sentirem grande melhora com o medicamento, desta forma a expectativa criada pelos jovens gera nos mesmos o anseio pela busca do conhecimento de forma rápida afim de irem bem nos estudos e tiveram boas notas sem se questionarem sobre os possíveis efeitos indesejados que podem aparecer a longo prazo.

Infelizmente nossa sociedade tem encontrado uma fonte de fácil acesso aos medicamentos, e essa problemática necessita ser efetivamente encarada pelos profissionais farmacêuticos. Nesse contexto é muito importante a orientação farmacêutica de forma que as informações sobre a terapêutica correta e os efeitos colaterais sobre esse fármaco seja passada de forma clara e objetiva para os jovens que queiram usar o fármaco somente para melhora das capacidades cognitivas.

Além da orientação farmacêutica apenas no balcão de uma farmácia ou drogaria, a criação de projetos que abordem o tema e que permitam a exploração ampliada dos conhecimentos do profissional farmacêutico sobre o assunto em universidades se torna imprescindível, pois desta forma as informações se consolidariam nos saberes adquiridos em sala de aula, possibilitando a formação de profissionais com conduta ética, uma visão crítica apurada, e ações transformadoras para sociedade.

\section{REFERÊNCIAS BIBLIOGRÁFICAS}


AFFONSO, R.S. et al. O uso indiscriminado do cloridrato de metilfenidato como estimulante por estudantes da área da Saúde da Faculdade Anhanguera de Brasília (FAB). Infarma Ciências Farmacêuticas, v. 28, n3. p. 166-172, out. 2016.

ANDRADE, L. S. et al. Ritalina uma droga que ameaça a inteligência. Revista de Medicina e Saúde de Brasília, v. 7, n. 1, p. 99-112, 2018.

BARROS, D.; ORTEGA, F. Metilfenidato e Aprimoramento Cognitivo Farmacológico: representações sociais de universitários. Saúde e Sociedade. São Paulo, v.20, n.2, p.350-362, 2011.

BRANT, L.C. CARVALHO, T.R.F. Metilfenidato: medicamento gadget da contemporaneidade. Interface, v. 16, n.42, jul./set. 2012.

BRASIL. Metilfenidato no tratamento de crianças com transtorno de déficit de atenção e hiperatividade, v. 8, n. 23, março, 2014. Disponível em:

$<$ http://portal.anvisa.gov.br/documents/33884/412285/Boletim+Brasileiro+de+Avalia\% C3\%A7\%C3\%A3o+de+Tecnologias+em+Sa\%C3\%BAde+\%28BRATS\%29+n\%C2\%B $\mathrm{A}+23 / \mathrm{fd} 71 \mathrm{~b} 822-8 \mathrm{c} 86-477 \mathrm{a}-9 \mathrm{f9d}-\mathrm{ac0c1d8b0187>}$. Acesso em 25 de mai. de 2019.

CALAZANS, A. G. C.; BELO, R. F. C. Prevalência do uso de metilfenidato por estudantes ingressantes nas universidades do município de Sete Lagoas/MG. Revista Brasileira de Ciências da Vida, v. 5, n. 1, 2017.

CALIMAN, L.V.; RODRIGUES, P. H. P. A. Experiência do uso de metilfenidato em adultos diagnosticados com TDAH. Psicologia em Estudo, Maringá, v. 19, n. 1, p. 125-134, jan./mar. 2014.

CARNEIRO, S.G. et al. O uso não prescrito de metilfenidato entre acadêmicos de Medicina. Ciências da Saúde e Biológicas. Rio de Janeiro, v. 8, n. 1, p. 53-59, abri/2013.

COLI, A. C. M.; SILVA, M. P. S.; NAKASU M. V. P. Uso não Prescrito de Metilfenidato entre Estudantes de uma Faculdade de Medicina do Sul de Minas Gerais. Revista Ciências em Saúde. Minas Gerais, v. 6, n. 3, 2016.

FARDIN C. E.; PILOTO, J. A. R. Uso indiscriminado do metilfenidato para o aperfeiçoamento cognitivo em indivíduos saudáveis. Revista UNINGÁ Review, v. 23, n. 3, p. 98-103, jul./set. 2015 .

FERNANDES, F.T. et al. Uso de substâncias psicoativas entre universitários brasileiros: perfil epidemiológico, contextos de uso e limitações metodológicas dos estudos. Cadernos saúde coletiva. Rio de Janeiro, v. 25 n. 4, p.498-507. 2017. 
FERNANDES, W. S.; CEMBRANELLI, J. C. Automedicação e o uso irracional de medicamentos: o papel do profissional farmacêutico no combate a essas práticas. Revista UNIVAP. São Paulo. v. 21, n. 37, jul., 2015.

GONÇALVES, C. S.; RIBEIRO, P. R. M. L. “Drogas da Inteligência?”: Cartografando as controvérsias do consumo da Ritalina para o aprimoramento cognitivo. Psicología, Conocimiento y Sociedad, Rio de Janeiro, v. 8, n.2, p. 71-94, set. 2018.

ITABORAHY, C. ORTEGA, F. O metifenidato no Brasil: uma década de publicações. Ciência \& Saúde Coletiva, Rio de Janeiro, v. 18, n.3, p, 803-816,2013.

JACOMINI, L.C.L. SILVA, N.A. Interações medicamentosas: uma contribuição para o uso racional de imunossupressores sintéticos e biológicos. Rev. Bras. Reumatol. São Paulo, v. 51, n. 2, mar./abr, 2011.

MONTEIRO, B.M.M.et al. Metilfenidato e melhoramento cognitivo em universitários: um estudo de revisão sistemática. SMAD, Revista Eletrônica Saúde Mental Álcool Drogas, Divinópolis, v. 13, n. 4 p. 232-242, out/dez, 2017.

MORGAN, H. L. et al. Consumo de Estimulantes Cerebrais por Estudantes de Medicina de uma Universidade do Extremo Sul do Brasil: Prevalência, Motivação e Efeitos Percebidos. Revista Brasileira de Educação Médica, Rio Grande Do Sul, p. 102-109, set., 2016.

MOTA, D. M. et al. Uso racional de medicamentos: uma abordagem econômica para tomada de decisões. Ciência Saúde Coletiva, Rio de Janeiro. v.13, jan, 2008.

MOURA, M.H. As consequências do uso prolongado e não terapêutico do metilfenidato. 2017. Trabalho de Conclusão de Curso (graduação em Farmácia,) Faculdade de Educação e Meio Ambiente, Rondônia.

NEGREIROS, A. C. D. R.; TELLES, M. V. L. Transtorno de Déficit de Atenção e Hiperatividade em crianças e adolescentes: revisão de literatura. Revista de Psicologia, v. 3, n.10, nov., 2009.

REIS, M. N. et al. Riscos associados ao uso inadequado do metilfenidato. Revista eletrônica saúde e ciência. Salvador, v. 8, n.2, nov., 2018.

SCHERER, L.; GUAZZELLI, C.T. Questões atuais sobre o uso da ritalina e sua relação com o ambiente escolar. Disponível em: < http://www.uniedu.sed.sc.gov.br/wpcontent/uploads/2016/02/Larissa-Scherer.pdf. >Acesso 30 de out. 2015.

SILVA, A. C. P. et al. A explosão do consumo de ritalina. Revista de Psicologia da UNESP, São Paulo, v. 11, n. 2, set., 2012. 
WILLE, A.R.F.; SALVI, J.O. Prevalência do uso de metilfenidato em acadêmicos de um centro universitário em ji-Paraná, Rondônia. Brazilian Journal of Surgery and Clinical Research - BJSCR, v. 24, n. 3, p. 13-19, set./nov., 2018. 